\title{
Laboratory At Distance: Importance of Student's Profile and Their Evaluated Experience
}

\author{
Vahé NERGUIZIAN ${ }^{I}$, Radhi MHIRI ${ }^{1}$, Jean-Sébastien DESCHÊNES ${ }^{2}$, Hamdjatou KANE ${ }^{3}$ and Maarouf SAAD \\ ${ }^{1}$ École de technologie supérieure, Montreal, Quebec, Canada \\ ${ }^{2}$ Université du Québec à Rimouski, Quebec, Canada \\ ${ }^{3}$ Université du Québec en Outaouais, Quebec, Canada \\ vahe.nerguizian@etsmtl.ca
}

\begin{abstract}
The paper presents the implementation and the outcomes of a new pedagogical active approach for laboratory work in electrical engineering at École de technologie supérieure (ÉTS) using interactive experiments. The objective was the introduction of Laboratory At Distance (LAD) executed by students. This approach considers communication via Internet through appropriate software programs with miniaturized kit connected to Personal Computer (PC) where work could be done from home or from any place (Lab@home). The specificities of the communication are designed to continually evolve with new feats of Information and Communication Technologies (ICT).

Judicious questionnaire for a survey was prepared to assess and validate the student's satisfaction with this approach. Satisfaction, dissatisfaction, suggestions and comments highlighted by students in the survey showed that student's profile is an important factor in bringing new approaches and suggested that the approach needs to be dynamic with necessary changes required during the application of LAD from one semester to another.
\end{abstract}

Keywords: Pedagogical approach: Lab@home: Miniaturized electronic kit: Analog electronic circuit: Information and Communications Technology (ICT).

\section{INTRODUCTION}

Several academic institutions developed pedagogical strategies to encourage and foster team collaborative work, ensuring simultaneous information exchanges between students themselves and with laboratory instructor or Professor. For the evaluation of different new pedagogical approaches, several active learning techniques were assessed, such as Problem Based Learning approach (PBL), Problem Project Based Approach (PPBL) by different researchers using new technological possibilities namely Information and Communication Technologies (ICT). These approaches were used for theoretical course periods, laboratory sessions and for tutorials.

Since ICT environments have become available, we have seen the development of a widespread use of numerical simulations for various scientific concepts and various technical processes. The simulation software allowed illustrating complex phenomena and friendly medium often used to test and understand various concepts. However, these solutions could not translate the physical reality of systems and did not allow the student direct contact with these systems. Moreover, the use of these simulations did not allow the development of practical skills that are particularly important in the case of scientific and technical training.

Laboratory work in an engineering curriculum is essential, but still, little is clear about what has to be done and how exactly one should bring the level of learning consolidated by the laboratory objectives $[1,2]$.

Remote laboratory is a tool/approach that tends to spread into science and engineering courses at different levels. It is used by several academic institutions to enrich science and engineering education by greatly expanding the range of experiments associated with the cognitive benefits on knowledge and competences of students.

For example, relative effectiveness of remote labs versus hands on labs in mechanical engineering course on machine dynamics and mechanisms was evaluated and the results were encouraging since about $90 \%$ of the student rated in favor to remote lab approach compared to handson lab [3].

With these positive outcomes of remote laboratory implementation at different institutions, the objective at École de technologie supérieure was the introduction of remote laboratory work with the availability of 2 types of platform. The first type considered remote laboratory work using laboratory equipment installed in the laboratory and connected via the Internet $[4,5]$. The second type considered work that could be performed with 
miniaturized equipment/kit connected to Personal Computer (PC) where the work could be even done from home [6].

The evolution of ICT and electronic technologies allowed us to consider a new extension of the laboratory to bring home (Lab@home). The idea was to take advantage of miniaturization and reduced cost of electronic kits, and facilities offered by the Personal Computer (PC). Student will have a miniature kit that includes basic electronics for the generation and processing of various types of signals.

Development of miniaturized and low cost various electronic devices, and further considerations of remote laboratory techniques to facilitate student's schedule originated the concept of home laboratory (Lab@home).

The main objective of Lab@home implementation is to consolidate the theoretical course material learned in class by experimental verification. In fact with the project based laboratory approach students are more concentrated and motivated to make the project work without realizing the importance of some basic concept thought in class. With the laboratory project approach, the student tends to cling to reality and often to the particular problem neglecting its generalized aspects. Moreover, the instructor has no more opportunity to ensure that the student deals efficiently with specific points in the laboratory activities. It is then necessary to provide activities that allow the student to check out some theoretical elements and discover relevant answers to questions related to the project. Lab@home supports the project by further parallel laboratory work using the concept of Mobile Laboratory with the help of ICT. It allows, during a short time laboratory, to explore a well-defined electronic circuit highlighting some theoretical concepts that the student discovered in his course lecture and giving her/him possible solutions for the project. Understanding experimentally some basic/specific and generalized concepts will help her/him perform better and succeed in her/his project completion.

At École de technologie supérieure (ÉTS), analog electronic circuit laboratory sessions were replaced by laboratory project based on project approach in 2006. The objectives of the project approach were to:

- Promote personal student engagement

- Arouse curiosity and stimulates the initiative of the student

- Create a situation where the student identifies himself with the project and puts all its efforts for a successful implementation

- Promote priority for knowledge integration

- Offer an opportunity in acquiring new knowledge and developing various engineering skills.

In 2012 and 2013, Mobile Laboratory or Lab@home was implemented on analog electronic circuit laboratory with different dynamic operation scenarios [6] to consolidate theoretical knowledge with experimental competences. Figure 1 shows the idea behind Lab@ home.

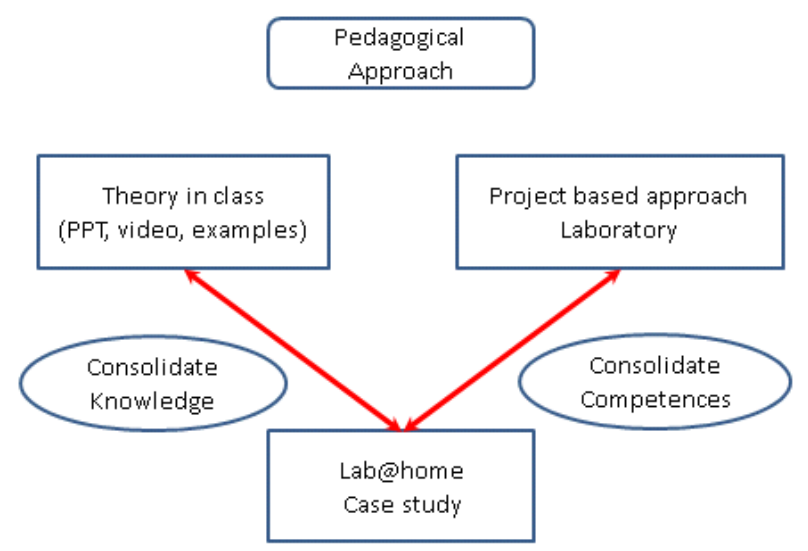

Fig. 1. Block diagram of Lab@home benefits

The LAD was implemented for day and evening classes with a total of 110 students over two semesters. Five different LADs were achieved by students associated to the course topics such as diodes, operational amplifiers and transistors.

Each LAD had the three learning steps: initial preparation steps (Pre-LAD), implementation of the experiment (LAD) and analysis/comment of the final results (Post-LAD) [6].

This article gives LAD implementation results and satisfaction feedback of students.

The paper is organized as follows: Section 2 describes the implementation and operation scenarios for each semester. Section 3 gives student's evaluation and survey results. Finally, discussions, conclusions and recommendations are presented in section 4 .

\section{LAB@HOME IMPLEMENTATION AND OPERATION}

\subsection{Operation scenarios}

The detailed description of Lab@home was described by the authors in [6] explaining the suggested implementation process with the advantages and the drawbacks of this approach. The details description (in French) of this approach with 5 different LADs called TLAD 1, 2, 3, 4 and 5 can be found on the Web site: http://tlad.etsmtl.ca/.

The implementation of this Lab@home approach started in fall 2012 semester with morning and evening classes of 80 students in total, followed by a morning class of 30 students in winter 2013 semester. From the beginning of the semesters, the miniaturized kit's operation guide and documentation about TLAD 
procedure were made available to the students on the Web site (Moodle) of the Mobile Laboratory. The TLAD instructor gave the students all working instructions. He clearly stated the importance of the 3 phase's TLAD steps for the success of TLAD experiments:

- Phase 1: The Pre-Lab@home, where student needs to get prepared for the experiments. This phase is very important for efficient time sharing between colleagues working on the same laboratory experiment. A lack of preparation could create a frustration between students and demotivation towards this approach blaming the complete process

- $\quad$ Phase 2: The Lab@ home, where student needs to do the experiments with kit present with her/him or kit present in another room, using ICT tools

- Phase 3: The Post-Lab@home, where student needs to write a report at the end of the experiments. At this phase a document will identify how the student will be evaluated and that information will reflect the description of the format of the final report.

\subsection{Fall 2012 Semester Scenarios}

Since the Lab@home was implemented the first time in the analog electronic course ELE200 at ÉTS, operation and implementation process was based with some cautions and considerations to avoid any technical difficulties that students can encounter during the semester. Five miniaturized kit (to accommodate all teams within the time frame of the laboratory) and two types of operation scenarios were used in fall semester:

- Scenario 1A: Each team of 2 students did one TLAD in the laboratory premises where they mounted all the electronic components on the miniaturized kit and got connected to the kit via Internet and TeamViewer software to complete their experiment. The operation was supported, if needed, by an instructor in the laboratory premises (Figure 2)

- Scenario 1B: Similarly the same teams did four TLADs in the laboratory premises where the miniaturized kit was at another location (in an assigned local) with all the electronic circuitry already mounted on the kit. Again students were connected to the kit via Internet and TeamViewer to complete their experiment. Another instructor was available in the local with the kit to give assistance at distance to students in need. In fact this instructor was even able to see the operation of all the four kits available to him (Figure 3).

The students of each team created a link/bridge via internet by TeamViewer with the password supplied by the instructor. For both process scenarios students did not see any perturbation during experiments. They followed properly the instructions given in Pre-Lab@home, Lab@home and Post-Lab@home.

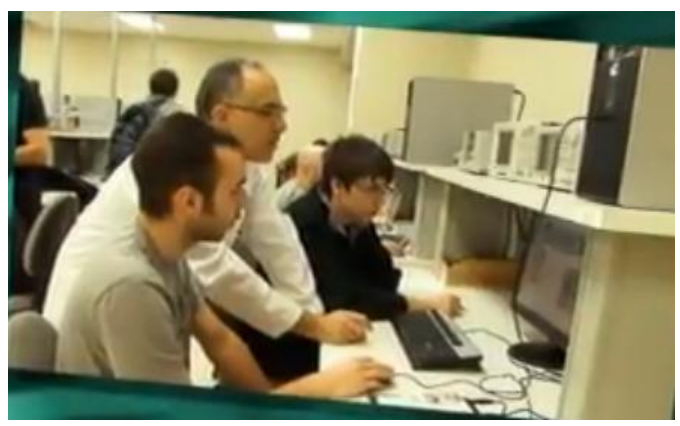

Fig. 2. Operation scenario $1 \mathrm{~A}$ where the miniaturized kit is available to the students in the laboratory location with a support by the instructor if needed.

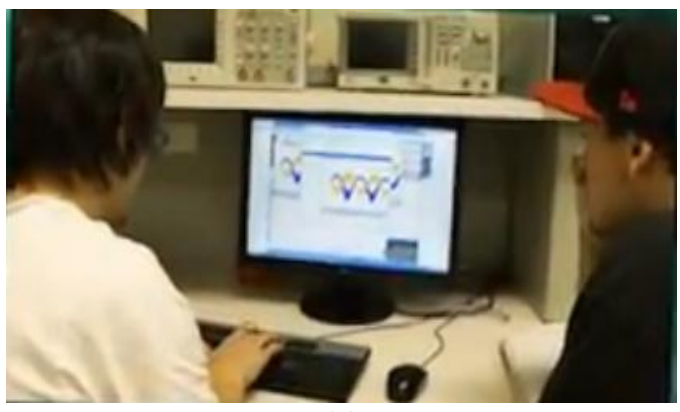

(a)

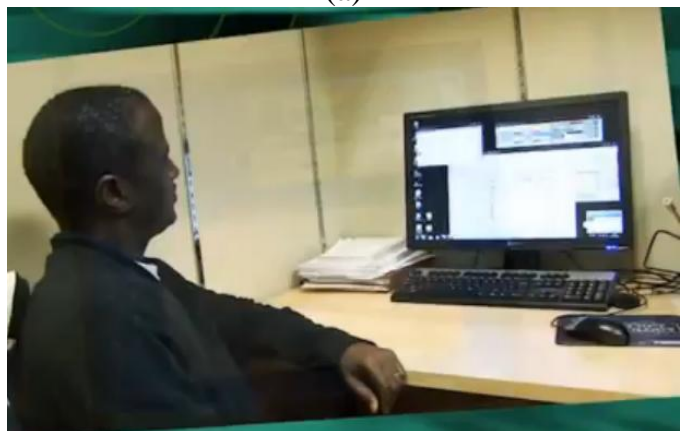

(b)

Fig. 3. Operation scenario 1B where the miniaturized kit is in another location not available to the students (a). The miniaturized kit at another location is supported dynamically by another instructor having access to all students operation (b).

\subsection{Winter 2013 Semester Scenarios}

For the winter semester two other types of operation scenarios were used:

- Scenario 2A:

Each student of a team, with its personal computer, was sharing with his colleague from distance the same kit located at ÉTS. The 
initiator of the experiment was connected to the kit via Internet using LogMeIn software with a password and time scheduling/allocation obtained by the instructor. Once connected to the kit, the initiator invited his/her colleague to share his/her computer through Join.me software with a specific password. The team was able to complete the 5 TLADs using this operation scenario (Figure 4)

- Scenario 2B:

Some students decided to be together in the same room (at home or another location) to have access at distant to the kit. This scenario was identical to $2 \mathrm{~A}$ without the need to use Join.me software (Figure 5).

With scenario 2A the student who was connected by Join.me to share the screen of TLAD initiator was having some minor delay problem. The delay was influencing the refreshing of the screen and delaying the response of any change made by the initiator. This was due to the additional access loading of Join.me software on LogMeIn. TeamViewer link of fall semester was replaced with LogMeIn and Join.me in winter semester since scheduling was difficult with TeamViewer.

Homemade software was used to schedule the access of each team with specified allocated time for each TLAD. Access schedule programming of TLAD experiments was made with the collaboration and consent of each team with the TLAD instructor.
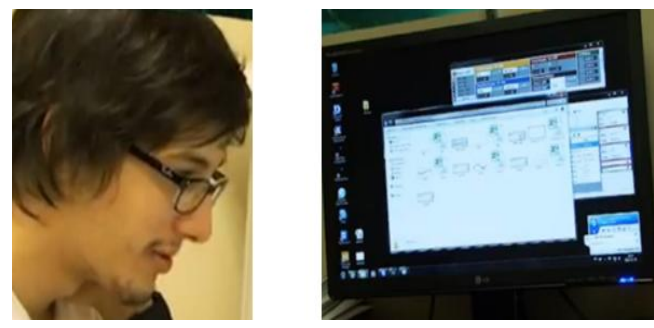

Fig. 3. Operation scenario $2 \mathrm{~A}$ where the miniaturized kit is at another location not available to the students and the initiator student shares his/her screen with his colleague.

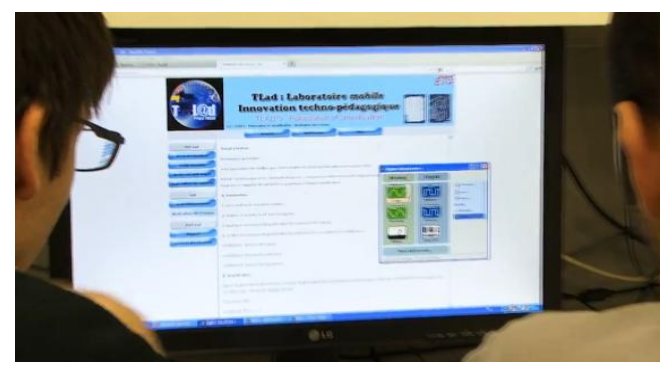

Fig. 4. Operation scenario 2B where the miniaturized kit is in another location not available to the students and both students of the team share the same screen during the experiment.

\section{STUDENT EVALUATION AND SURVEY}

During both semesters the feedback of student was probed by talking with them in classes and asking them to respond sincerely to a prepared survey with several questions related to TLAD experiments.

Survey results for fall semester 2012 are capture in Table 1 after references section. The results are presented in the format of 'not at all agreed' to 'strongly agreed' with their percentage. The comments given by students were related to contextualization of the work and the time allocated during the experiments.

Survey of winter 2013 showed quiet similar results with less student's satisfaction than fall semester.

\section{DISCUSSIONS, CONCLUSIONS AND RECOMMENDATIONS}

The implementation and the operation of the TLAD experiments were a dynamic process requiring a process change from one session to the other to satisfy student's needs and to enhance the overall process. Some comments received by students initiated LAD operation modification from one semester to the other.

At the beginning of LAD implementation (Scenarios $1 \mathrm{~A}$ and $1 \mathrm{~B})$ student's supervision was high avoiding any unexpected technical problem and supporting students for any unanswered questions. In fall semester no serious technical problems were observed for LAD operation. Therefore in winter 2013, it was decided to modify the process (scenarios $2 \mathrm{~A}$ and $2 \mathrm{~B}$ ) and let the students become more independent and autonomous. This modification reflected exactly Lab@home operation process where the students were doing their experiments from home.

The survey completed by students in fall semester showed that they are satisfied with the process except that TLAD experiments were more time demanding. For winter semester student found that TLAD experiments were too much time demanding and they needed some explanation and support after each TLAD experiment. This is why the survey results were less satisfactory.

Student's comments and recommendation were taken into account and it was decided to evaluate the profile of all students at every semester to adapt the operation by minor modifications and enhancement for better results and effectiveness. Therefore, students profiling (course loading, number of hours per week of employment, family situation, etc.) will be continuously monitored for next coming semesters and adjustment made accordingly. Moreover new survey questions will be added to get more detailed feedback from students.

CEEA13; Paper 091

Montreal, QC; June 17-20, 2013 


\section{Acknowledgements}

The authors would like to acknowledge the support of FODAR (Fonds de développement académique du réseau de l'université du Québec) and of École de technologie supérieure.

\section{References}

[1] Feisel, L. D., Rosa, A. J., Journal of Engineering Education 2005, 94, 121-130.

[2] Salinger, G. L., Journal of Technology Studies 2005, 31, 5.

[3] Corter, J. E., J. V. Nickerson, et al. (2004). Remote versus hands-on labs: A comparative study. Frontiers in Education, 2004. FIE 2004. 34th Annual, IEEE.

[4] Mhiri, R., Saad, M., Nerguizian, V., Amadou, M. SaliahHassane, H., Ouertani, S., Sahli, S., Brady, G., La technopédagogie dans les travaux de laboratoire contribue à un meilleur apprentissage et davantage de motivation en science et en technologie, Association Internationale de Pédagogie Universitaire (AIPU 2012), Trois- Rivières, Quebec, Canada, 14-18 May, 2012

[5] Radhi Mhiri, Maarouf Saad, Moustapha Dodo Amadou, Gérald Brady, Saber Ouertani, Sandra Sahli, Hamadou Saliah-Hassane, Vahé Nerguizian, The experience of a collaborative project on remote laboratory: From development to operation, IEEE International Conference on E-Learning in Industrial Electronics, (ICELIE 2012), October 25-28, 2012, Montreal, Quebec, Canada.

[6] Vahé Nerguizian, Radhi Mhiri, Hamdjatou Kane, JeanSébastien Deschênes, Hamadou Saliah-Hassane, Maarouf Saad, Lab@home for analog electronic circuit laboratory, IEEE International Conference on E-Learning in Industrial Electronics, (ICELIE 2012), October 25-28, 2012, Montreal, Quebec, Canada.

Table 1: Survey results of fall 2012 semester with morning and evening classes of 80 students

\begin{tabular}{|c|c|c|c|c|c|}
\hline $\begin{array}{l}\text { Indicate on a scale of } 1 \text { to } 4 \text { how much you agree with the following statements } \\
\text { ( } 1=\text { not at all agreed } \ldots . .4=\text { strongly agreed) }\end{array}$ & 1 in $\%$ & $2 \mathrm{in} \%$ & 3 in $\%$ & 4 in $\%$ & $3+4$ in $\%$ \\
\hline $\begin{array}{l}\text { You are a teacher of ELE } 200 \text { and we suggest you to introduce } \\
\text { TLAD experiments in this course. } \\
\text { Will you support this idea? (* indicate your suggestions) }\end{array}$ & 0 & 28 & 62 & 10 & 72 \\
\hline The TLAD were used to support the classroom learning & 0 & 22 & 60 & 18 & 78 \\
\hline $\begin{array}{l}\text { The TLAD allowed me to discover new technical knowledge } \\
\text { (FFT analysis system, etc. ...) }\end{array}$ & 0 & 12 & 56 & 32 & 88 \\
\hline $\begin{array}{l}\text { Contextualization of the work (problem description) in TLAD } \\
\text { are important and interesting }\end{array}$ & 13 & 32 & 23 & 32 & 55 \\
\hline The time allocated for TLAD is sufficient & 8 & 21 & 21 & 50 & 71 \\
\hline Overall I consider TLAD a successful experience & 0 & 28 & 48 & 24 & 72 \\
\hline I would prefer to achieve the TLAD from home & 0 & 20 & 50 & 30 & 80 \\
\hline $\begin{array}{l}\text { Instructions and information given on the Web site of TLAD } \\
\text { are sufficient to achieve TLAD experiments }\end{array}$ & 9 & 13 & 46 & 32 & 78 \\
\hline At the end of this semester, did you appreciate more the TLAD experiments? & 0 & 41 & 41 & 18 & 59 \\
\hline
\end{tabular}

